INFLUENCIA DE LA GLOBALIZACIÓN TECNOLÓGICA EN LA CAPACIDAD OPERATIVA DEL PUERTO DE MANZANILLO.

\title{
INFLUENCIA DE LA GLOBALIZACIÓN TECNOLÓGICA EN LA CAPACIDAD OPERATIVA DEL PUERTO DE MANZANILLO
}

\section{TECHNOLOGICAL GLOBALIZATION INFLUENCE ON OPERATIONAL CAPACITIES IN THE PORT OF MANZANILLO}

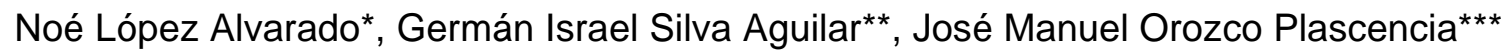

*Maestro en Derecho Aduanal. Facultad de Comercio Exterior de la Universidad de Colima. ORCID: https://orcid.org/0000-0001-8908-1828.

**Doctor en Ciencias Administrativas y Negocios Internacionales. Facultad de Comercio Exterior de la Universidad de Colima. Email: german_s@ucol.mx. ORCID: https://orcid.org/0000-0002-5966-1845.

***Doctor en Relaciones Transpacíficas. Facultad de Economía de la Universidad de Colima. Email: manuel_orozco@ucol.mx. ORCID: https://orcid.org/0000-0002-8878-2996.

Dirección para recibir correspondencia: noelopez@ucol.mx 
INFLUENCIA DE LA GLOBALIZACIÓN TECNOLÓGICA EN LA CAPACIDAD OPERATIVA DEL PUERTO DE MANZANILLO.

\section{RESUMEN}

OBJETIVO: Identificar y analizar la influencia que ha ejercido la globalización tecnológica, en la capacidad del Puerto marítimo de Manzanillo.

MATERIAL Y MÉTODO: La investigación es de tipo analítica descriptiva, ya que se sustenta fundamentalmente en información recabada de análisis realizados por diversas instituciones y fuentes secundarias que permiten conceptualizar ambas variables.

RESULTADOS: El mantenimiento de los servicios ya establecidos en el Puerto de Manzanillo es meramente importante para el aumento en las relaciones comerciales, estas dos variables aumentan de manera casi equivalente, a mayor inversión en el mantenimiento, mayor movimiento de carga contenerizada.

CONCLUSIONES: La inversión pública a nivel estatal para la infraestructura en el Puerto de Manzanillo resulta ser buena para el incremento en el movimiento de TEU; sin embargo, ésta inversión resulta ser más tangible y constante para el mantenimiento de la infraestructura ya establecida en el Puerto, lo cual tiene un fuerte impacto.

PALABRAS CLAVE: Globalización tecnológica. Capacidad. Desempeño económico y comercial. 
INFLUENCIA DE LA GLOBALIZACIÓN TECNOLÓGICA EN LA CAPACIDAD OPERATIVA DEL PUERTO DE MANZANILLO.

\section{ABSTRACT}

OBJECTIVE: To identify and analyze the influence of technological globallization on operational capacities in the Port of Mazanillo.

MATERIAL AND METHOD: The following is an analytical descriptive research, based on information collected through content analysis performed by several institutions and also from secondary sources in order to conceptualize both variables.

RESULTS: The maintenance of the established services in the Port of Manzanillo is crucial in order to increase commeercial relations, these two variables increase almost equally, the more investment in the maintenance, the more movement of cargo in containers.

CONCLUSIONS: Public investment for the port of Manzanillo infrastructure at a state level turns out to be good for increasing the movement of TEU; however, this investment turns out to be more tangible and constant for the maintenance of the established infrastructure in the Port, thus having a great impact.

KEY WORDS: Technological globalization. Capacity. Economic and commercial performance. 
INFLUENCIA DE LA GLOBALIZACIÓN TECNOLÓGICA EN LA CAPACIDAD OPERATIVA DEL PUERTO DE MANZANILLO.

\section{INTRODUCCIÓN}

Actualmente, el proceso de globalización tecnológica representa no solo una transformación a la mayoría de las industrias, sino un reto y simultáneamente una oportunidad de crecimiento para todos los países que se adapten y aprovechen los cambios que conlleve dicha transformación. El comercio exterior hoy en día, es uno de los sectores que sufre diversas y constantes adaptaciones a los cambios que surgen a partir de las nuevas necesidades de la sociedad; es decir, es una industria que tiene como principal prioridad atender de forma eficaz las nuevas coyunturas comerciales; tal y como es el caso de los Puertos de México y específicamente del Puerto de Manzanillo, Colima; el cual se posiciona como el principal vínculo en el Pacífico, pues permite establecer grandes conexiones comerciales.

El objetivo principal de este trabajo de investigación, es analizar la capacidad del Puerto de Manzanillo en función de la influencia de la globalización tecnológica del 2010 al 2017; es decir, describir el desarrollo y transformación que ha sufrido el Puerto, gracias a la intervención de los factores globalizadores.

El desarrollo de esta investigación está encaminado, primeramente a establecer los antecedentes del problema, factor que permite la delimitación correcta del mismo; después, se presenta la justificación de la investigación, planteando al mismo tiempo las variables e hipótesis. Posteriormente, se hace un marco teórico conceptual de la literatura y se establece un marco contextual que permite conocer las situaciones actuales. Por último, se presentan los resultados y conclusiones de la investigación.

El proceso evolutivo de la humanidad, ha dado origen a un grado de integración e interdependencia entre las naciones y es a partir de la Revolución Industrial cuando se puede hablar del término "Globalización"; sin embargo, dicho término se enfoca de acuerdo al ámbito de su aplicación, por ende, esta investigación se centraliza en la incidencia de la globalización tecnológica dentro la capacidad portuaria; por ello, es sustancial dar inicio a este proyecto con el concepto general de la globalización y la tecnología.

De acuerdo con Rodolfo Cerdas (1940), la globalización se define como "el acelerado proceso de cambio que, a nivel mundial, se ha venido desarrollando en todos los ámbitos del quehacer humano, pero muy particularmente en lo referente a lo militar, lo económico, el comercio, las finanzas, la información, la ciencia, la tecnología, el arte y la cultura" (s. p.). 
INFLUENCIA DE LA GLOBALIZACIÓN TECNOLÓGICA EN LA CAPACIDAD OPERATIVA DEL PUERTO DE MANZANILLO.

Por otra parte, la tecnología se entiende como:

El medio a través del cual se traslada el conocimiento científico a la solución de problemas concretos de una manera efectiva. De allí, la tendencia de valorar a las ciencias en términos de lo que aportan a la sociedad. Tecnología es crear competencias y se expresa en entidades tecnológicas que consisten en aparatos, procedimientos y habilidades (Van Wyk, 2004, citado por Zartha y Herrera, 2009, p. 2).

Es decir, la tecnología "permite extender el alcance de la actividad humana, en todo ámbito organizacional, ya sea producción, comercialización, distribución, uso o consumo de bienes y servicios" (Zartha y Herrera, 2009, p. 3). Por consiguiente, a partir de ambas definiciones se entiende por globalización tecnológica al proceso que sufre cualquier ente sometido bajo a un aporte de cambio que se genera a partir de la implementación de un conocimiento o técnica que tenga como prioridad el mejoramiento del mismo.

En cuanto a la capacidad portuaria es un término que hoy en día ha tomado mayor participación dentro de la esfera económica comercial; ya que, el nivel de interconexión entre los países ha incrementado en la última década debido precisamente al impacto de la globalización. Sin embargo, es complicado medir de manera general cómo se ha dado dicho impacto pues de acuerdo con la reflexión de Serrano y Trujillo (2005) "no es conveniente analizar el Puerto globalmente porque es una organización compleja, cada parte con objetivos y niveles de eficiencia distintos, así que es preferible analizar una actividad concreta" (p. 16).

La presente investigación se delimita de manera espacial en México, específicamente en el Puerto marítimo de Manzanillo en el Estado de Colima; en dicha área geográfica es donde se enfoca el proyecto. En relación con la temporalidad, es un estudio longitudinal que abarca desde los años 2007 hasta el año en curso y hace uso de cifras y datos obtenidos por diversas instituciones oficiales cómo la Secretaría de Economía (SE), el Instituto Nacional de Estadística y Geografía (INEGI), la Comisión Económica para América Latina y el Caribe (CEPAL), la Conferencia de las Naciones Unidas sobre Comercio y Desarrollo (UNCTAD), entre otras.

En el sentido estricto de la investigación, el problema se orienta al estudio cualitativo de la influencia de la globalización tecnológica en la actividad portuaria de Manzanillo; ya que, se analiza el desempeño comercial del mismo dentro de los sectores económicos a nivel nacional e internacional; es decir, se estudia la influencia que ha tenido la globalización tecnológica en el 
INFLUENCIA DE LA GLOBALIZACIÓN TECNOLÓGICA EN LA CAPACIDAD OPERATIVA DEL PUERTO DE MANZANILLO.

cambio de procesos, actividades y desarrollo comercial del Puerto y que ha permitido la creación de ventajas competitivas a través de la implementación de nuevas técnicas y conocimiento otorgados a partir del cambio tecnológico debido a la globalización.

En la última década, la actividad y la capacidad portuaria de México, se ha visto modificada en su funcionamiento, participación, e integración, dentro del comercio exterior; ya que, como se ha expuesto con anterioridad la globalización tecnológica es factor fundamental que permite incrementar los niveles de conectividad y competitividad. Por ende, a partir de la implementación de un conjunto de avances tecnológicos se puede contextualizar en qué medida o cómo impacta dicho fenómeno globalizador en el Puerto de Manzanillo, Colima.

Debido a ello, encontrar los efectos que ejerce la globalización tecnológica dentro del comportamiento competitivo del Puerto de Manzanillo, se considera de gran relevancia, ya que comúnmente se relaciona el nivel de participación comercial con el grado de apertura de un Puerto.

En ese sentido, la presente investigación pretende analizar de qué manera ha influido la globalización tecnológica en la capacidad portuaria, relacionando dicho termino con el movimiento de carga TEU en el Puerto de Manzanillo, pues de acuerdo con Mesino (2009) "el conocimiento es hoy la principal ventaja competitiva de las naciones" (p. 137).

Las variables de la investigación son las siguientes:

Variable independiente (X): Globalización Tecnológica.

Variable dependiente (Y): Capacidad Portuaria. 
INFLUENCIA DE LA GLOBALIZACIÓN TECNOLÓGICA EN LA CAPACIDAD OPERATIVA DEL PUERTO DE MANZANILLO.

\section{Tabla 1}

Matriz de congruencia

Pregunta general $\begin{aligned} & \text { Preguntas } \\ & \text { específicas }\end{aligned} \quad$ Hipótesis general Objetivo general Objetivos específicos

\begin{tabular}{|c|c|c|c|c|}
\hline $\begin{array}{l}\text { ¿La globalización } \\
\text { tecnológica } \\
\text { influye positiva y } \\
\text { directamente en } \\
\text { la capacidad del } \\
\text { Puerto de } \\
\text { manzanillo? }\end{array}$ & $\begin{array}{l}\text { 1.- ¿Cuál es el } \\
\text { nivel de } \\
\text { conectividad del } \\
\text { Puerto marítimo } \\
\text { de Manzanillo a } \\
\text { partir de la } \\
\text { innovación? } \\
\text { 2.- ¿Cómo ha } \\
\text { mejorado la } \\
\text { infraestructura } \\
\text { portuaria a partir } \\
\text { de la inversión } \\
\text { privada? }\end{array}$ & $\begin{array}{l}\text { La globalización } \\
\text { tecnológica } \\
\text { influye positiva y } \\
\text { directamente en } \\
\text { la capacidad del } \\
\text { Puerto marítimo } \\
\text { de Manzanillo. }\end{array}$ & $\begin{array}{l}\text { Analizar la } \\
\text { capacidad del } \\
\text { Puerto marítimo } \\
\text { de Manzanillo en } \\
\text { función de la } \\
\text { influencia de la } \\
\text { globalización } \\
\text { tecnológica del } \\
2010 \text { al } 2017 .\end{array}$ & $\begin{array}{l}\text { 1.- Analizar y evaluar el } \\
\text { nivel de conectividad del } \\
\text { Puerto marítimo de } \\
\text { Manzanillo a partir de la } \\
\text { innovación } \\
\text { 2.- Describir y evaluar la } \\
\text { infraestructura portuaria } \\
\text { a partir de la inversión } \\
\text { privada. }\end{array}$ \\
\hline
\end{tabular}

Fuente: Elaboración propia.

A continuación, se presentarán los principales aspectos que serán objeto de búsqueda y análisis a través de la investigación:

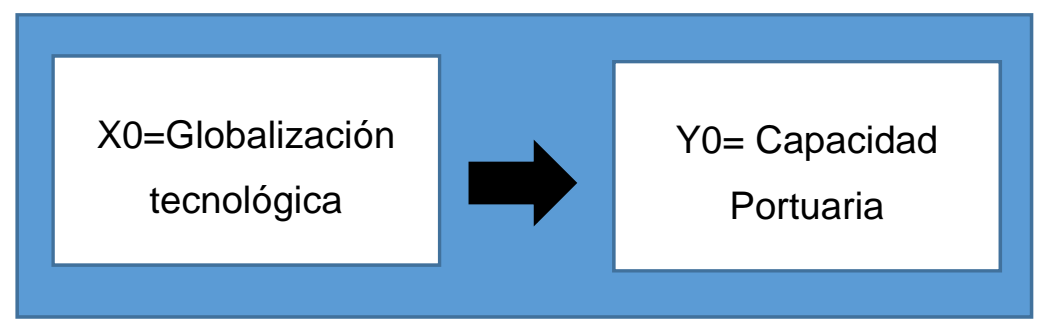

Figura 1. Despliegue de las variables de investigación.

Fuente: Elaboración propia. 
INFLUENCIA DE LA GLOBALIZACIÓN TECNOLÓGICA EN LA CAPACIDAD OPERATIVA DEL PUERTO DE MANZANILLO.

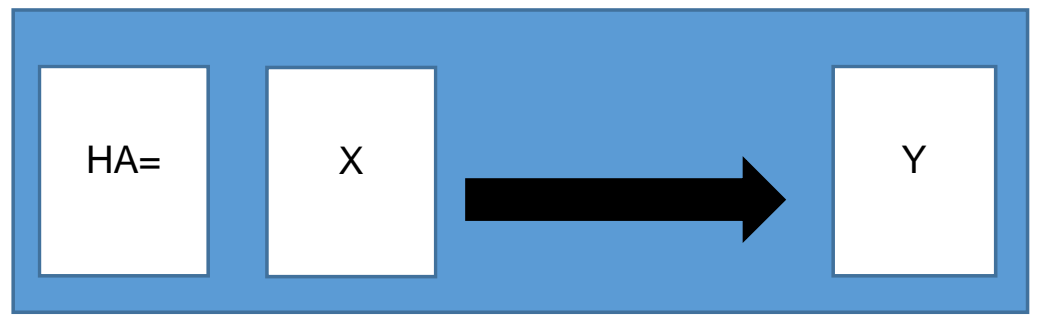

Figura 2. Despliegue de las variables de investigación.

Fuente: Elaboración propia.

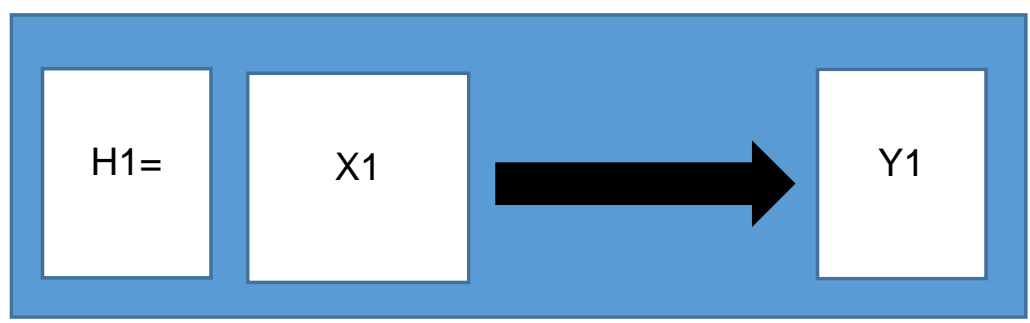

Figura 3. Despliegue de las variables de investigación.

Fuente: Elaboración propia.

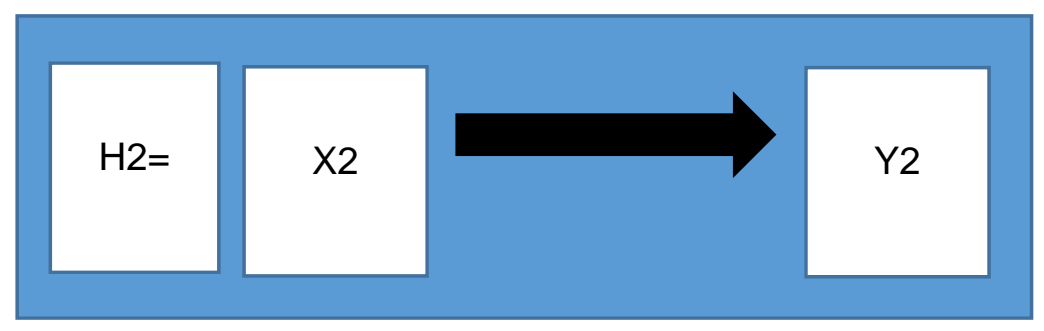

Figura 4. Despliegue de las variables de investigación.

Fuente: Elaboración propia.

Las relaciones anteriores, representan los campos de estudio, observación y medición, que se tomarán en cuenta para realizar esta investigación. Contando también con la descripción de las variables dependiente e independiente, lo que sustenta la relación directa entre las dimensiones de cada una. 
INFLUENCIA DE LA GLOBALIZACIÓN TECNOLÓGICA EN LA CAPACIDAD OPERATIVA DEL PUERTO DE MANZANILLO.

\section{Tabla 2}

Despliegue de las variables de investigación

\begin{tabular}{|c|c|c|}
\hline Variables & Descripción de la variable & Dimensiones \\
\hline $\begin{array}{c}\text { X0 } \\
\text { Globalización } \\
\text { Tecnológica }\end{array}$ & $\begin{array}{l}\text { Se entiende, como: "el fenómeno experimentado por el mundo de la } \\
\text { invención y la innovación". También, al retomar este término se } \\
\text { puede hacer referencia a la "tecnoglobalización" que de acuerdo con } \\
\text { Alejandro Rojo (2015) se define como, "la difusión de la tecnología a } \\
\text { través del mundo, de las culturas, de las naciones; atravesando } \\
\text { fronteras" (Rojo, 2015). }\end{array}$ & $\begin{array}{l}\text { X2. Inversión } \\
\text { privada }\end{array}$ \\
\hline $\begin{array}{c}\text { YO } \\
\text { Capacidad } \\
\text { Portuaria }\end{array}$ & $\begin{array}{l}\text { La capacidad portuaria se define, como "el volumen de tráfico que el } \\
\text { puerto puede atender en el periodo de tiempo de un año (medida en } \\
\text { toneladas por año). Ahora bien, la capacidad del puerto va a ser en } \\
\text { función del ritmo de llegada de los buques, del ritmo de } \\
\text { carga/descarga, de la longitud y calado de los muelles, del área de } \\
\text { almacenamiento de la mercancía y del ritmo de llegada de camiones } \\
\text { o trenes (ritmo de evacuación de mercancía), entre otros factores" } \\
\text { (Gil, 2007, p. 15). }\end{array}$ & $\begin{array}{c}\text { Y2. } \\
\text { Infraestructura } \\
\text { portuaria }\end{array}$ \\
\hline
\end{tabular}

Fuente: Elaboración propia.

Para definir el término de "globalización tecnológica" es importante mencionar que tal y como se ha tratado anteriormente éste se refiere a un proceso global, por lo cual infiere en ámbitos culturales, sociales, políticos y económicos. Por lo que, dentro de éste apartado están conceptos generales del término, pero enfocados al área tecnológica.

Ulrich Beck sostiene que "con la globalización, en todas sus dimensiones, surge frente a esto, no sólo una nueva multiplicidad de conexiones y relaciones entre Estados y sociedades, sino que además se arraiga con mayor fuerza todavía la estructura de los presupuestos fundamentales según los cuales se ideaban, organizaban y vivían hasta ahora las sociedades y los Estados en cuanto a unidades territoriales recíprocamente delimitadas. La globalidad quiere decir, que se rompe la unidad del estado nacional y de la sociedad nacional, y se establecen unas relaciones nuevas de poder y competitividad, unos conflictos y entrecruzamientos entre, por una parte, unidades y actores del mismo estado nacional y, por la otra, actores, identidades, espacios, situaciones y procesos sociales trasnacionales" (Beck, 2008, p. 58). 
INFLUENCIA DE LA GLOBALIZACIÓN TECNOLÓGICA EN LA CAPACIDAD OPERATIVA DEL PUERTO DE MANZANILLO.

De acuerdo con Guillermo, "La globalización se puede definir como un proceso dinámico de creciente libertad e integración mundial de los mercados de trabajo, bienes, servicios, tecnología y capital" (De la Dehesa, 2007, p. 19).

Para Patrick Fourgeaud (2000), es la capacidad portuaria "depende del porcentaje de carga del buque, de los movimientos no productivos (como posicionamiento de la carga), del nivel de automatización de las grúas, del peso medio de la unidad de carga y del porcentaje de mercancía que requiere una especial atención, y además del número de diferentes compañías que operan en una misma terminal" (Fourgeaud, 2000, citado por Gil, 2017, p. 16).
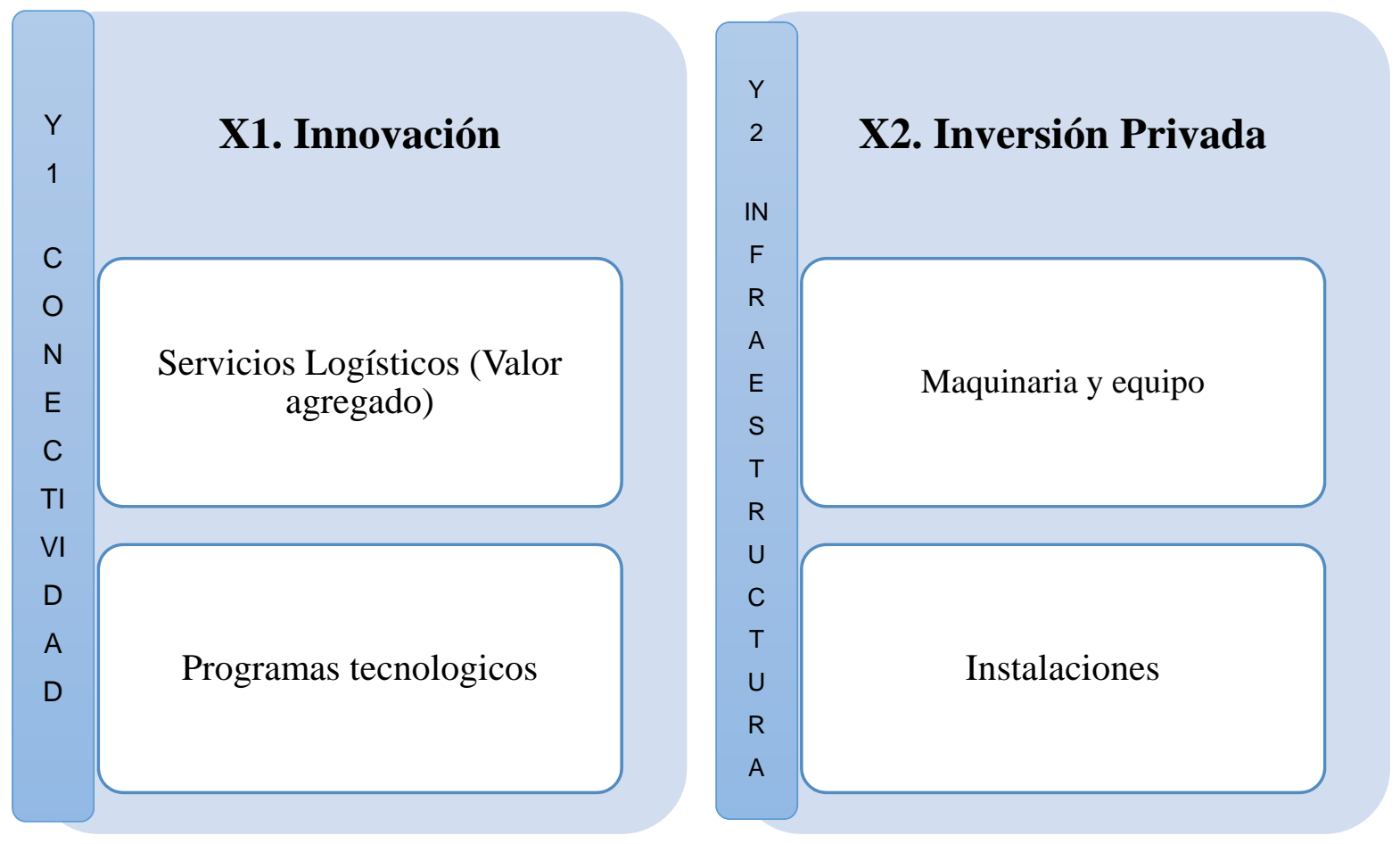

Figura 5. Relación de dimensiones y operación.

Fuente: Elaboración propia. 
INFLUENCIA DE LA GLOBALIZACIÓN TECNOLÓGICA EN LA CAPACIDAD OPERATIVA DEL PUERTO DE MANZANILLO.

\section{Tabla 3}

Teorías

\begin{tabular}{|c|c|c|}
\hline Autores & Nombre de la teoría & Principales postulados \\
\hline $\begin{array}{l}\text { Robert Solow } \\
\text { y Trevor W. } \\
\text { Swan (1956) }\end{array}$ & $\begin{array}{l}\text { Teoría neoclásica del } \\
\text { crecimiento. }\end{array}$ & $\begin{array}{l}\text { "Pretende predecir el desarrollo económico de un país a largo plazo (...), } \\
\text { esta teoría se centra en cómo tres factores (tecnología, capital y } \\
\text { envergadura de la mano de obra) impulsan el crecimiento económico" } \\
\text { (Marron, } 2011, \text { p. } 70 \text { ). }\end{array}$ \\
\hline \multirow[t]{2}{*}{ Paul Romer } & $\begin{array}{l}\text { Nueva teoría del } \\
\text { crecimiento, también } \\
\text { conocida como teoría } \\
\text { del crecimiento } \\
\text { endógeno. }\end{array}$ & $\begin{array}{l}\text { "El crecimiento económico se da siempre que las personas toman } \\
\text { recursos y reestructuran haciéndolos más valiosos (...) no obstante, la } \\
\text { historia del ser humano nos enseña que el crecimiento económico nace } \\
\text { de las mejores recetas, no sólo de más cocción" (Romer, s. f.: s. p., } \\
\text { citado por Marron, } 2011, \text { p. } 70 \text { ). }\end{array}$ \\
\hline & & $\begin{array}{l}\text { "Sugiere que los gobiernos deberían dedicarse a fomentar la inversión en } \\
\text { innovación. Deberían proporcionar un entorno que la haga posible, } \\
\text { construyendo un sistema de propiedad intelectual bien estructurado" } \\
\text { (Marron, 2011, p. 72). }\end{array}$ \\
\hline $\begin{array}{l}\text { Fitoussi } \\
(1997)\end{array}$ & $\begin{array}{l}\text { Capacidad de la } \\
\text { globalización. }\end{array}$ & $\begin{array}{l}\text { "la globalización separa cada vez más a los países o personas que se } \\
\text { adaptan a la nueva situación y las nuevas tecnologías y a aquellos que } \\
\text { no son capaces de hacerlo" (Fitoussi, 1997, s. p, citado por De la } \\
\text { Dehesa, 2007, p. 91). }\end{array}$ \\
\hline $\begin{array}{l}\text { Michael Porter } \\
(1990)\end{array}$ & $\begin{array}{l}\text { La ventaja } \\
\text { competitiva. }\end{array}$ & $\begin{array}{l}\text { "La prosperidad de una nación depende de su competitividad, la cual se } \\
\text { basa en la productividad con la cual ésta produce bienes y servicios. } \\
\text { Políticas macroeconómicas e instituciones legales sólidas y políticas } \\
\text { estables, son condiciones necesarias, pero no suficientes para asegurar } \\
\text { una economía próspera. La competitividad está fundamentada en las } \\
\text { bases microeconómicas de una nación: la sofisticación de las } \\
\text { operaciones y estrategias de una compañía y la calidad del ambiente } \\
\text { microeconómico de los negocios en la cual las compañías compiten." } \\
\text { (Porter, 1990, s. p, mencionado por González, Zamora, Celaya, Navarro, } \\
2016, \text { p. 32). }\end{array}$ \\
\hline \multirow[t]{3}{*}{$\begin{array}{l}\text { Eliyahu } \\
\text { Goldratt, } 1984\end{array}$} & $\begin{array}{l}\text { Teoría de las } \\
\text { restricciones. }\end{array}$ & $\begin{array}{l}\text { Goldratt, 1995, plantea que: "es necesario el empleo continuado de la } \\
\text { dirección de las limitaciones, lo que consiste en principios de } \\
\text { razonamientos sistemáticos, permitiendo la identificación de los } \\
\text { problemas de fondo, la construcción de soluciones válidas y completas y } \\
\text { la creación de planes de implantación inmunes." }\end{array}$ \\
\hline & & $\begin{array}{l}\text { Uno de los conceptos fundamentales de la Teoría de Restricciones es la } \\
\text { analogía entre los sistemas complejos y las cadenas: la cadena completa } \\
\text { no es más resistente que su eslabón más débil (la restricción del } \\
\text { sistema). La gestión por restricciones intenta explotar el potencial del } \\
\text { sistema total a través de la gerencia de sus eslabones más débiles. }\end{array}$ \\
\hline & & $\begin{array}{l}\text { La interrelación existente entre las actividades logísticas y entre éstas y } \\
\text { las áreas de la empresa, nos conducen a entender la existencia de un } \\
\text { sistema integrado. En este sistema, cada actividad requiere de una } \\
\text { adecuada coordinación para: optimizar el funcionamiento del proceso de } \\
\text { negocios y reducir costos. }\end{array}$ \\
\hline
\end{tabular}

Fuente: Elaboración propia. 
INFLUENCIA DE LA GLOBALIZACIÓN TECNOLÓGICA EN LA CAPACIDAD OPERATIVA DEL PUERTO DE MANZANILLO.

\section{Tabla 4}

Revisión de la literatura

\begin{tabular}{cll}
\hline Nombre & Autor y año & \multicolumn{1}{c}{ Aportación } \\
\hline Just in time (JIT)- Toyotismo $\quad$ Taiichi Ohno, 1976. & $\begin{array}{l}\text { Sistema de gestión estratégica que busca } \\
\text { fomentar relaciones estables de } \\
\text { aprovisionamiento y agruparlas a pocos } \\
\text { proveedores que cuenten con un alto nivel } \\
\text { de experiencia y conocimiento, logrando } \\
\text { asína reducción de costos y un alto } \\
\text { funcionamiento competitivo, todo esto se } \\
\text { entiende como unidad de valor } \\
\text { diferenciador ante la satisfacción del } \\
\text { cliente. }\end{array}$
\end{tabular}

Fuente: Elaboración propia.

\section{MATERIAL Y MÉTODO}

Esta investigación es de tipo hemerográfica y bibliográfica; ya que, para su realización se llevan a cabo búsquedas de teorías y conceptos en revistas científicas y fuentes secundarias.

También es de tipo cualitativa y analítica, debido a que a partir de la información recabada se analizan los datos y posteriormente se realiza una interpretación de los mismos. 
INFLUENCIA DE LA GLOBALIZACIÓN TECNOLÓGICA EN LA CAPACIDAD OPERATIVA DEL PUERTO DE MANZANILLO.

\section{Tabla 5}

Diseño de la investigación

\begin{tabular}{|c|c|c|c|c|}
\hline Variables & Descripción de la variable & Dimensiones & $\begin{array}{l}\text { Instrumento de } \\
\text { la investigación }\end{array}$ & $\begin{array}{c}\text { Operacionalización de } \\
\text { variables }\end{array}$ \\
\hline $\begin{array}{c}\text { X0 } \\
\text { Globalización } \\
\text { Tecnológica }\end{array}$ & $\begin{array}{l}\text { Se entiende, como: "el } \\
\text { fenómeno experimentado } \\
\text { por el mundo de la } \\
\text { invención y la innovación". } \\
\text { También, al retomar este } \\
\text { término se puede hacer } \\
\text { referencia a la } \\
\text { "tecnoglobalización" que de } \\
\text { acuerdo con Alejandro Rojo } \\
\text { (2015) se define como, "la } \\
\text { difusión de la tecnología a } \\
\text { través del mundo, de las } \\
\text { culturas, de las naciones; } \\
\text { atravesando fronteras" } \\
\text { (Rojo, 2015). }\end{array}$ & $\begin{array}{l}\text { X1. } \\
\text { Innovación } \\
\text { X2. Inversión } \\
\text { privada }\end{array}$ & $\begin{array}{l}\text { Análisis } \\
\text { hemerográfico } \\
\text { y bibliográfico. }\end{array}$ & $\begin{array}{l}\text { Sistemas tecnológicos } \\
\text { que han mejorado el } \\
\text { funcionamiento del } \\
\text { puerto e incrementan } \\
\text { su competitividad. } \\
\text {-Automatización del } \\
\text { Puerto. }\end{array}$ \\
\hline $\begin{array}{c}\text { YO } \\
\text { Capacidad } \\
\text { Portuaria }\end{array}$ & $\begin{array}{l}\text { La capacidad portuaria se } \\
\text { define, como "el volumen de } \\
\text { tráfico que el puerto puede } \\
\text { atender en el periodo de } \\
\text { tiempo de un año (medida } \\
\text { en toneladas por año). } \\
\text { Ahora bien, la capacidad } \\
\text { del Puerto va a ser en } \\
\text { función del ritmo de llegada } \\
\text { de los buques, del ritmo de } \\
\text { carga/descarga, de la } \\
\text { longitud y calado de los } \\
\text { muelles, del área de } \\
\text { almacenamiento de la } \\
\text { mercancía y del ritmo de } \\
\text { llegada de camiones o } \\
\text { trenes (ritmo de evacuación } \\
\text { de mercancía), entre otros } \\
\text { factores" (Gil, 2017, p. 15). }\end{array}$ & $\begin{array}{c}\text { Y1. } \\
\text { Conectividad } \\
\\
\text { Y2. } \\
\text { Infraestructura } \\
\text { portuaria }\end{array}$ & $\begin{array}{l}\text { Análisis } \\
\text { hemerográfico } \\
\text { y bibliográfico. }\end{array}$ & $\begin{array}{l}\text {-Ampliaciones del } \\
\text { Puerto. } \\
\text {-Mejoramiento de la } \\
\text { infraestructura a través } \\
\text { de la inversión privada. } \\
\text {-Maquinaria y equipo. }\end{array}$ \\
\hline
\end{tabular}

Fuente: Elaboración propia. 
INFLUENCIA DE LA GLOBALIZACIÓN TECNOLÓGICA EN LA CAPACIDAD OPERATIVA DEL PUERTO DE MANZANILLO.

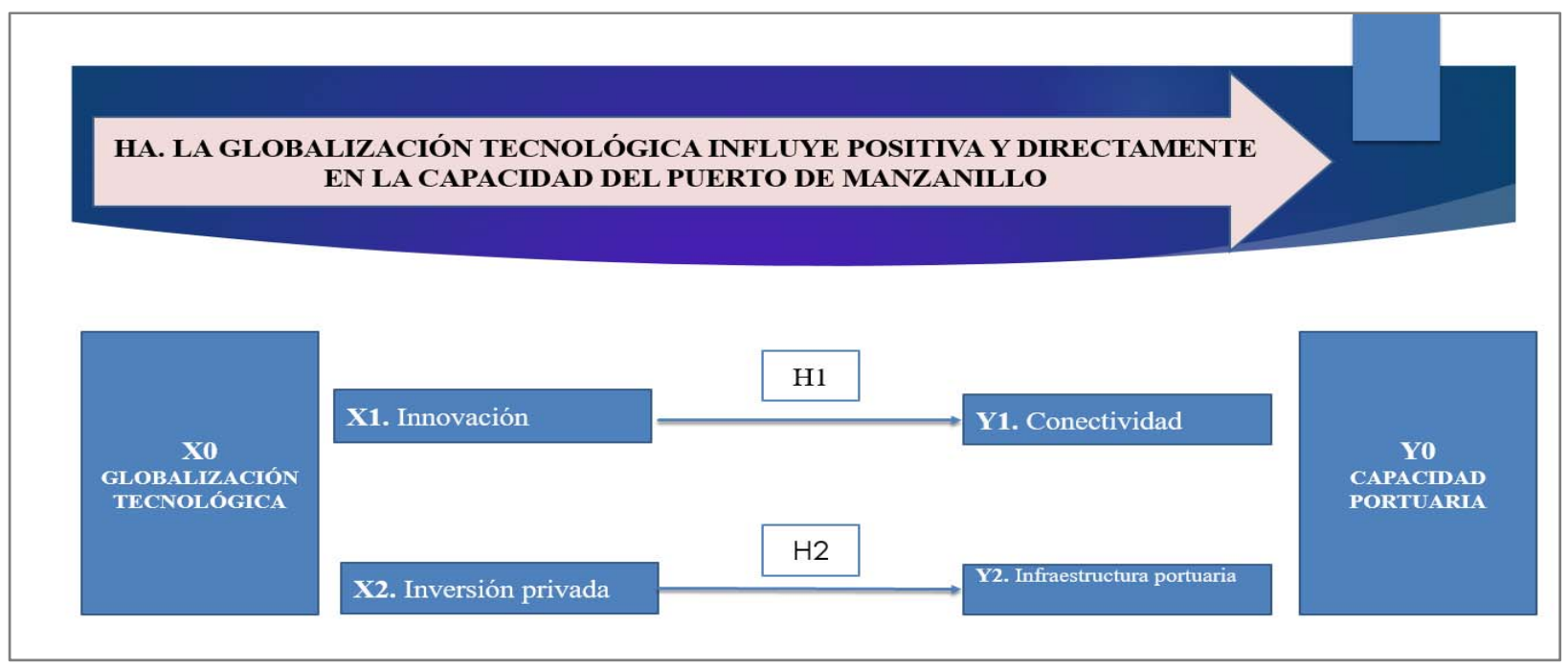

Figura 6. Constructo de investigación.

Fuente: Elaboración propia.

Se emplean como instrumentos de investigación el análisis y revisión de artículos, y libros relacionados con el tema de estudio.

Para la realización de este proyecto se consultó de manera amplia el contenido de la Secretaría de Economía (SE), CEPAL, UNCTAD, Administración Portuaria Integral (API), fuentes que brindan alto grado de veracidad a la información que se retoma a partir de sus estudios.

Según el ranking de Movimiento de Contenedores de CEPAL (2017), se sitúa al Puerto de Manzanillo como el $4^{\circ}$ lugar, y arroja las cifras exactas de movimiento contenerizado de los años a analizar, las cuales son:

Tabla 6

Cifras en millones de acuerdo al movimiento contenerizado

\begin{tabular}{lccc}
\hline Año & 2014 & 2015 & 2016 \\
\hline Movimiento Contenerizado (millones) & $2,355,149$ & $2,541,140$ & $2,578,822$ \\
\hline
\end{tabular}

Fuente: Elaboración propia con base en cifras de CEPAL. 
INFLUENCIA DE LA GLOBALIZACIÓN TECNOLÓGICA EN LA CAPACIDAD OPERATIVA DEL PUERTO DE MANZANILLO.

Cifras que serán comparadas con otras variables según el modelo cronométrico creado, para relacionar la dimensión de infraestructura.

Modelo Cronométrico:

Movimiento Contenerizado en el Puerto de Manzanillo (Millones) $=+$ (Inversión anual en infraestructura, Costos y Gastos Anuales por Infraestructura).

\section{VARIABLES}

- Movimiento Contenerizado: Adquiere el lugar de "X" variable dependiente, ya que se analiza, que tanta relación tienen las siguientes variables para que esta incremente, o decremente según su dependencia con la variable de Infraestructura. Esta variable hace referencia a los Millones de TEU en movimiento según el año.

- Inversión Anual en Infraestructura: Esta variable ocupa el lugar de variable independiente o "Y". Hace referencia la inversión pública a nivel Estado que se invierte en el Puerto de Manzanillo anualmente, para mejorar, actualizar, o ampliar las instalaciones del Puerto.

- Costos y Gastos Anuales por Infraestructura: También ocupando el lugar de variable independiente o "Y", esta variable indica la inversión en Millones de pesos mexicanos que se destinan al mantenimiento de las instalaciones, infraestructura y/o servicios del Puerto de Manzanillo. Dichas variables serán comparadas entre sí, la variable " $X$ " se compara con cada una de las variables " $Y$ " en un análisis individual por cada una de ellas.

La SCT (2018) menciona las cifras en inversión anual por infraestructura millones de pesos, incluyendo muelles, urbanización, obras de protección, dragados, entre otros, los cuales ocupan la primera variable de "Y" que se analiza: 
INFLUENCIA DE LA GLOBALIZACIÓN TECNOLÓGICA EN LA CAPACIDAD OPERATIVA DEL PUERTO DE MANZANILLO.

Tabla 7

Inversión pública anual en infraestructura

\begin{tabular}{cccc}
\hline Año & 2014 & 2015 & 2016 \\
\hline $\begin{array}{c}\text { Inversión Pública anual en } \\
\text { infraestructura }\end{array}$ & $301,000,000$ & $111,000,000$ & 0 \\
\hline
\end{tabular}

Fuente: Elaboración propia, basada en información de SCT.

Se puede observar, que en el año 2016 según SCT (2018) es nula, por lo cual se prevé que el resultado será negativo, con un nivel de correlación considerable para el movimiento de carga contenerizada, a continuación, se presenta el análisis.

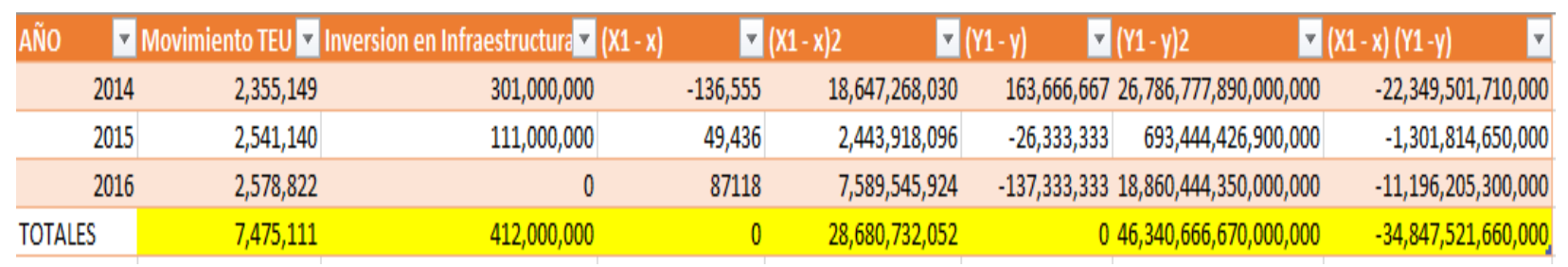

\begin{tabular}{|l|r|}
\hline DATOS & VALOR \\
\hline $\mathrm{n}$ & 3 \\
\hline $\mathrm{x}$ & $2,491,704$ \\
\hline $\mathrm{y}$ & $137,333,333$ \\
\hline Covarianza & $-17,423,760,830,000$ \\
\hline Desviacion Estandar (X1) & $119,751.27$ \\
\hline Desviacion Estandar (Y1) & $152,218,045.40$ \\
\hline Coef. De Correlacion & -1.911725917 \\
\hline
\end{tabular}

Figura 7. Análisis.

Fuente: Elaboración propia.

Claramente se observa como la Covarianza resulta negativa, por ende nuestro coeficiente de correlación será meramente negativo, lo cual dice que la relación de las variables " $X$ " (Movimiento TEU) con la variable "Y" (Inversión anual en Infraestructura), actúa de manera contraria, esto quiere decir que mientras una incrementa otra, decrementa.

La desviación estándar de dichas variables, indica que tan lejos están los puntos de la media (Covarianza), se observa cómo el valor "Y" es más grande que el de "X"; por lo tanto, sus puntos son muy dispersos de la media o covarianza. 
INFLUENCIA DE LA GLOBALIZACIÓN TECNOLÓGICA EN LA CAPACIDAD OPERATIVA DEL PUERTO DE MANZANILLO.

Finalmente, se puede analizar el coeficiente de correlación, notando que la relación es mayor a 1 , lo cual indica que su relación es muy fuerte, pero que no aumentan al mismo ritmo, ya que la correlación es negativa, esto tiene un significado más profundo, pues no quiere decir que con la inversión en infraestructura, la carga contenerizada decremente, si no que de acuerdo a los datos en el año 2016, según SCT no hubo inversión pública en el Puerto, pero años previos al 2016 si se invirtió, lo cual hizo que la carga contenerizada aumentara, aunque no de manera considerable, ya que, si bien es observado que en el año 2016 se tiene el mayor movimiento de carga contenerizada de los años a analizar; según CEPAL, en el 2018 se cortó de tajo la inversión una vez que la carga aumentó, es por eso que nuestra correlación es muy fuerte, pero negativa, lo cual deja ver la gran importancia de la inversión de los gobiernos en infraestructura para el aumento en el volumen de transporte en las TEU.

La SCT (2018) arroja las cifras exactas de los años a analizar en cuanto a costos y gastos anuales por infraestructura en millones de pesos mexicanos, la cual incluye Administración, Mantenimiento de Obras de Atraque y Muelle, Mantenimiento de Señalamiento Marítimo, Mantenimiento de Área de Urbanización, Mantenimiento de Servicios Logísticos, entre otros. Dicha variable se compara con la variable principal " $X$ " o la variable de movimiento de carga contenerizada.

Tabla 8

Costos y gastos en infraestructura

\begin{tabular}{lccc}
\hline Año & 2014 & 2015 & 2016 \\
\hline $\begin{array}{l}\text { Costos y gastos } \\
\begin{array}{l}\text { anuales en } \\
\text { infraestructura }\end{array}\end{array}$ & $194,400,000$ & $195,100,000$ & $259,000,000$ \\
\hline
\end{tabular}

Fuente: Elaboración propia, basada en SCT.

Se observa como la inversión en los años a analizar ha sido constante, por lo que se espera que su impacto en la variable " $X$ " o movimiento de carga contenerizada tenga un impacto positivo en el análisis. A continuación, se presentan los resultados de dicho análisis: 
INFLUENCIA DE LA GLOBALIZACIÓN TECNOLÓGICA EN LA CAPACIDAD OPERATIVA DEL PUERTO DE MANZANILLO.

\begin{tabular}{|c|c|c|c|c|c|c|c|}
\hline AÑo & \multicolumn{2}{|c|}{$\nabla$ Movimiento TEU $\nabla$ Costos y Gastos en Infraesti) $(X 1-x)$} & \multicolumn{2}{|c|}{$\nabla(x 1-x) 2$} & $(y 1-y)$ & $\nabla(Y 1-y) 2$ & $x)(Y 1-y)$ \\
\hline 2014 & $2,355,149$ & $194,400,000$ & $-136,555$ & $18,647,268,030$ & $-21,766,666,70$ & $70 \quad 473,787,779,200,000$ & $2,972,347,171,000$ \\
\hline 2015 & $2,541,140$ & $195,100,000$ & 49,436 & $2,443,918,096$ & $-21,066,666.70$ & $70 \quad 443,804,445,800,000$ & $1,041,451,735,000$ \\
\hline 2016 & $2,578,822$ & $259,000,000$ & 87,118 & $7,589,545,924$ & 42,833 & $331,834,694,442,000,000$ & $3,731,554,330,000$ \\
\hline TALES & $7,475,111$ & $648,500,000$ & 0 & $28,680,732,052$ & & $0 \quad 2,752,286,667,000,000$ & $7,745,353,236,000$ \\
\hline
\end{tabular}

\begin{tabular}{|l|r|}
\hline DATOS & V VALOR \\
\hline $\mathrm{n}$ & 3 \\
\hline $\mathrm{X}$ & $2,491,704$ \\
\hline $\mathrm{y}$ & $216,166,666.70$ \\
\hline Covarianza & $3,872,676,618,000$ \\
\hline Desviacion Estandar (X1) & $119,751.27$ \\
\hline Desviacion Estandar (Y1) & $37,096,405.94$ \\
\hline Coef. De Correlación & 0.871764701 \\
\hline
\end{tabular}

Figura 8. Análisis.

Fuente: Elaboración propia.

Se observa, como la covarianza de las variables resulta ser positiva, lo cual nos dice que, la relación entre ellas es positiva, y que las dos incrementan en conjunto. Mientras tanto, la desviación estándar de cada una presenta una gran diferencia en las cifras, lo cual dice que sus puntos a la media son dispersos.

Finalmente, el coeficiente de correlación se presenta de manera positiva y fuerte, lo cual se interpreta, la inversión en costos y gastos es bien aprovechada para el movimiento de las TEU, el mantenimiento de los servicios ya establecidos en el Puerto de Manzanillo es meramente importante para el aumento en las relaciones comerciales, estas dos variables aumentan de manera casi equivalente, a mayor inversión en el mantenimiento, mayor movimiento de carga contenerizada.

\section{CONCLUSIONES}

La inversión pública a nivel estatal para la infraestructura en el Puerto de Manzanillo, resulta ser buena para el incremento en el movimiento de TEU; sin embargo, esta inversión resulta ser más tangible y constante para el mantenimiento de la infraestructura ya establecida en el Puerto, lo cual tiene un fuerte impacto. Sin embargo, la correlación entre el movimiento de las TEU es aún más fuerte con la inversión en infraestructura que, aunque resulta ser negativa es mucho más fuerte, lo cual indica que se debe invertir más en ampliar el puerto, pero de manera constante para que de esta manera sea una relación fuerte y positiva, de igual manera mantener las instalaciones de manera adecuada, tal como se vio en los años analizados para 
INFLUENCIA DE LA GLOBALIZACIÓN TECNOLÓGICA EN LA CAPACIDAD OPERATIVA DEL PUERTO DE MANZANILLO.

que la inversión en infraestructura pueda tener un impacto positivo, y con el mantenimiento de ella, se aumente de manera aún más significativa el movimiento de carga contenerizada. Por ende, se concluye que la variable de infraestructura resulta ser una de las más influyentes dentro del aumento en la carga contenerizada en el Puerto de Manzanillo, por lo cual, debe ser atendida de manera constante.

Finalmente, se concluye por comprobar satisfactoriamente la hipótesis que se plantea dentro de esta investigación; ya que, de acuerdo con el análisis y comprensión de la información estudiada, se entiende que desde que surgió el término globalización, se ha generado un fuerte impacto dentro de diferentes ámbitos, como: el económico y tecnológico, restructurando así el nivel de desarrollo de las naciones.

En el caso particular de este proyecto y tomando en cuenta el Puerto de Manzanillo como unidad de estudio, se entiende que el grado de inferencia de la globalización tecnológica ha modificado positivamente su nivel de capacidad portuaria; es decir, ha incrementado su competitividad comercial, ya que, hoy en día, el Puerto de Manzanillo es considerado como el principal vínculo fortalecido en el Pacífico dedicado para las grandes transacciones comerciales, no sólo por la gran concentración empresarial, sino porque gracias a la inversión dedicada a su infraestructura se ha habilitado el aumento de su capacidad de carga, factor que le permite participar activamente dentro del comercio exterior.

Se observa, que de acuerdo a los análisis presentados, es indispensable mantener una constante inversión en el mantenimiento de la infraestructura, servicios logísticos, obras de atraque, señalamientos, que hagan del Puerto de Manzanillo una terminal con mayores índices de apertura y competitividad comercial.

\section{REFERENCIAS BIBLIOGRÁFICAS}

Administración Portuaria Integral de Manzanillo (2018). Infraestructura. Consultado el 13 de julio de 2018. Recuperado de https://www.puertomanzanillo.com.mx/esps/0000203/infraestructura

Beck, U. (2008). ¿Qué es la globalización? Falacias del globalismo, respuestas a la globalización. Barcelona, España: Paidós.

De la Dehesa, G. (2007). Comprender la globalización. Madrid, España: Alianza. 
INFLUENCIA DE LA GLOBALIZACIÓN TECNOLÓGICA EN LA CAPACIDAD OPERATIVA DEL PUERTO DE MANZANILLO.

Comisión Económica para América Latina y el Caribe (2017). Los puertos mexicanos que figuran en el ranking 2017 de la CEPAL. Recuperado de http://t21.com.mx/maritimo/2018/05/29/ puertos -mexicanos-que-figuran-ranking-2017-cepal

Gil, C. (2007). Definición de los niveles de servicio de las terminales portuarias. Consultado el 13 de julio 2018. Recuperado de https://upcommons.upc.edu/bitstream/handle/2099.

1/5770/04.pdf?sequence=5\&isAllowed=y

Google (2017). Mapa satelital de México. Consultado el 15 de julio del 2018. Recuperado de https://mapas.viajesyfotos.net/satelitales/mexico.html

Marron, D. (2011). 50 teorías económicas sugerentes y desafiantes. Barcelona, España: BLUME.

Mesino, L. (2009). La globalización económica y sus implicaciones socio-culturales en América Latina. Revista de Ciencias Sociales (Ve), 15(1), pp. 126-138.

Morales, A. (1999). Globalización: conceptos, características y contradicciones, Reflexiones, 78(1), s. p.

Rojo, A. (2015). La tecnoglobalización. Blogspot. Consultado el 13 de julio 2018. Recuperado de http://latecnoglobalizacion.blogspot.com/

Secretaría de Comunicaciones y Transportes (2018). Puertos mexicanos con capacidad mundial. Consultado el 13 de julio del 2018. Recuperado de https://www.gob.mx/sct/articulos/puertos-mexicanos-con-capacidad-mundial162214?idiom=es

Zartha, J. y Herrera, J. (2009). Nociones, ciencia, tecnología, innovación. Bolivia: Alianza estratégica entre el Departamento Administrativo de Ciencia, Tecnología e Innovación Colciencias. 